\title{
A new method to identify subclasses among AGB stars using Gaia and 2MASS photometry
}

\author{
T. Lebzelter ${ }^{1}$, N. Mowlavi ${ }^{2}$, P. Marigo ${ }^{3}$, G. Pastorelli ${ }^{3}$, M. Trabucchi ${ }^{3}$, P. R. Wood ${ }^{4}$, and I. Lecoeur-Taïbi ${ }^{2}$ \\ 1 University of Vienna, Department of Astrophysics, Tuerkenschanzstrasse 17, 1180 Vienna, Austria \\ e-mail: thomas.1ebzelter@univie.ac.at \\ 2 Department of Astronomy, University of Geneva, Ch. des Maillettes 51, 1290 Versoix, Switzerland \\ 3 Dipartimento di Fisica e Astronomia Galileo Galilei, Università di Padova, Vicolo dell'Osservatorio 3, 35122 Padova, Italy \\ ${ }^{4}$ Research School of Astronomy and Astrophysics, Australian National University, Canberra, ACT 2611, Australia
}

Received 11 June 2018 / Accepted 10 August 2018

\begin{abstract}
Aims. We explore the wealth of high-quality photometric data provided by data release 2 (DR2) of the Gaia mission for long-period variables (LPVs) in the Large Magellanic Cloud (LMC). Our goal is to identify stars of various types and masses along the asymptotic giant branch.

Methods. For this endeavour, we developed a new multi-band approach combining Wesenheit functions $W_{\mathrm{RP}, \mathrm{BP}-\mathrm{RP}}$ and $W_{K_{\mathrm{s}}, J-K_{\mathrm{s}}}$ in the Gaia BP, RP, and 2MASS $J, K_{\mathrm{s}}$ spectral ranges, respectively, and use a new diagram, $\left(W_{\mathrm{RP}, \mathrm{BP}-\mathrm{RP}}-W_{K_{\mathrm{s}}, J-K_{\mathrm{s}}}\right)$ versus $K_{\mathrm{s}}$, to distinguish between different kinds of stars in our sample of LPVs. We used stellar population synthesis models to validate our approach.

Results. We demonstrate the ability of the new diagram to discriminate between O- and C-rich objects, and to identify low-mass, intermediate-mass, and massive O-rich red giants, as well as extreme C-rich stars. Stellar evolution and population synthesis models guide the interpretation of the results, highlighting the diagnostic power of the new tool to discriminate between stellar initial masses, chemical properties, and evolutionary stages.
\end{abstract}

Key words. stars: AGB and post-AGB - stars: evolution - stars: variables: general - Magellanic Clouds

\section{Introduction}

Asymptotic giant branch (AGB) stars represent a decisive part within the evolution of low- and intermediate-mass stars. These high-luminosity, cool objects contribute significantly to the metal content of their host galaxy by various nucleosynthesis processes in their interiors, deep mixing events, and strong mass loss. The first two points modify the surface composition - even changing the chemistry from O-rich to C-rich - which affects the mass loss. All these contributions depend on the stellar parameters, in particular the stars' masses.

To study the role of AGB stars for the evolution of galaxies, it is important to reliably identify and characterize (mass, chemistry) them within a stellar population. AGB stars show a typical variability pattern with long periods of between several tens of to several hundred days and moderate to large light amplitudes, in particular in the visual range. These variables form the class of the long-period variables (LPVs) which also includes other cool, evolved stars like red supergiants or stars at the tip of the red giant branch (RGB). This kind of variability is easily detectable even in other galaxies and therefore LPVs define an excellent sample of AGB stars.

Major advances in the understanding of AGB stars were often related to large surveys in various photometric bands. Monitoring surveys like MACHO (Wood et al. 1999) and OGLE (e.g., Soszyński et al. 2009) and others delivered excellent samples of AGB stars for various galaxies and the Galactic bulge. With the advent of the Gaia mission (Gaia Collaboration 2016), a new era is starting. The all-sky nature of the Gaia survey; the high spatial resolution of $\sim 0.4$ arcsec; the provision of parallaxes; the availability of three photometric bands providing wide $G$, blue $G_{\mathrm{BP}}$, and red $G_{\mathrm{RP}}$ magnitudes for more than 1.5 billion stars, from very bright (few magnitudes in $G$ ) to as faint as $G \simeq 20.7$ mag; and the provision of time series in all three bands for all the stars, not mentioning the expected provision of radial velocity and astrophysical parameters for stars brighter than $G \simeq 16 \mathrm{mag}$, are some of the unique advantages of the survey.

In Gaia data release 2 (DR2, Gaia Collaboration 2018), the first Gaia catalog of LPVs with $G$-band variability amplitudes larger than $0.2 \mathrm{mag}$ (measured by the $5-95 \%$ quantile range of the $G$ time series) has been published (Mowlavi et al. 2018). It contains 151761 candidates. As a first step in the analysis of this unique dataset, we study in this Letter the Gaia DR2 LPVs in the Large Magellanic Cloud (LMC).

The above mentioned task of identification and distinction of various groups of stars among the LPVs is challenging because the upper giant branch represents a mixture of objects of various masses and evolutionary stages. In this Letter we present a new method for an efficient identification of stars of different mass and chemistry based on Gaia and 2MASS photometry.

\section{LPV characterization using Gaia and 2MASS photometry}

The most efficient way to distinguish between various types of stars in large samples, like that of an entire galaxy, is via 
photometry. Frequently used in this context is the distinction between O-rich and C-rich stars by using near-infrared (NIR) photometry $\left(J-K_{\mathrm{s}}\right)$ from the 2MASS all sky catalogue (Skrutskie et al. 2006). Cross-matches within a search radius of 1 " between Gaia and 2MASS sky positions were obtained for all the 11022 Gaia LMC LPVs studied here (see below for the selection of LMC candidates), except for only seven candidates.

The $K_{\mathrm{s}}$-band is a relatively good representative of the bolometric magnitude for red giants. To obtain a reddening free magnitude, we used the NIR Wesenheit function $W_{K, J-K}=$ $K_{\mathrm{s}}-0.686 \cdot(J-K)$ (in the following $W_{K}$, see Soszynski et al. 2005). Several authors have already used $W_{K}$, for example, for the construction of period-luminosity diagrams of LPVs (e.g., Trabucchi et al. 2017).

The intrinsic color spread of red giant stars being relatively small in the IR - except for large values of circumstellar reddening when gas and dust expelled from the star surround the object -, the Wesenheit function $W_{K}$ can adequately correct for interstellar reddening using $J-K_{\mathrm{s}}$, since interstellar reddening impacts both the $K_{\mathrm{s}}$ magnitude and the $J-K_{\mathrm{s}}$ color.

In the visual range, the color of red giants is very sensitive to the surface temperature, and there is a large intrinsic visual color spread in a population of red giants. This is particularly the case for the Gaia $G_{\mathrm{BP}}-G_{\mathrm{RP}}$ color that we use in this study, since $G_{\mathrm{BP}}-G_{\mathrm{RP}}$ is strongly affected by the temperature sensitivity of molecules dominating these wavelength ranges. The molecules present in the atmosphere depend on the $\mathrm{C} / \mathrm{O}$ ratio. Therefore, a degeneracy exists between interstellar reddening, circumstellar reddening, chemistry, and temperature differences at a given luminosity. By using Wesenheit functions, we can eliminate the interstellar reddening component (and much of the circumstellar reddening component) from this degeneracy.

We now define the Wesenheit function $W_{\mathrm{RP}}$, a reddening-free combination of $G_{\mathrm{BP}}$ and $G_{\mathrm{RP}}$ magnitudes. The factor multiplying $\left(G_{\mathrm{BP}}-G_{\mathrm{RP}}\right)$ in $W_{\mathrm{RP}}$ can be computed using the interstellar extinction data provided by Schlegel et al. (1998), adapted for the $\mathrm{BP}$ and RP filter curves. As a result ${ }^{1}$, we get

$W_{\mathrm{RP}}=G_{\mathrm{RP}}-1.3 \cdot\left(G_{\mathrm{BP}}-G_{\mathrm{RP}}\right)$.

In LPVs, $W_{\mathrm{RP}}$ combines temperature, chemistry (O- or C-rich), reddening, and brightness information. This is not the case for $W_{K}$, the color $J-K_{\mathrm{s}}$ being much less sensitive to the surface temperatures and chemistry of LPVs than $G_{\mathrm{BP}}-G_{\mathrm{RP}}$ is. We return to this point below.

In a next step we attempted to combine the three quantities $K_{\mathrm{s}}, W_{\mathrm{RP}}$, and $W_{K}$ in a two-dimensional (2D) diagram by combining $W_{\mathrm{RP}}$ and $W_{K}$. In Fig. 1 we plot $W_{\mathrm{RP}}-W_{K}$ on the $x$-axis and $K_{\mathrm{s}}$ on the $y$-axis for all Gaia DR2 LPVs identified in the LMC. The selection of members is based on sky position $\left(50^{\circ}<\alpha<105^{\circ}\right.$ and $\left.-77^{\circ}<\delta<-61^{\circ}\right)$, proper motion $\left(1.2<\mu_{\alpha}^{*}<2.5\right.$ and $-0.8<\mu_{\delta}<1.5$, in mas $\mathrm{yr}^{-1}$ ), and parallaxes $(\varpi<0.5 \mathrm{mas})$, which results in $11022 \mathrm{LMC}$ candidates. As described in more detail in Appendix A, it is important to understand that Fig. 1 is not a color-magnitude diagram (CMD) in the classical sense since the bluest objects are located in the middle of the diagram, around $W_{\mathrm{RP}}-W_{K}=0.8 \mathrm{mag}$ for the LMC, and the objects are getting redder towards both sides of the diagram. This is illustrated, for the left side of the diagram, in Fig. 1 where the color-coding of the points shows that the stars have an increasing $G_{\mathrm{BP}}-G_{\mathrm{RP}}$ color (they become redder) when $W_{\mathrm{RP}}-W_{K}$

\footnotetext{
1 We use the median values of $G_{\mathrm{BP}}$ and $G_{\mathrm{RP}}$ provided in gaiadr2.vari_time_series_statistics at https://gea.esac . esa.int/archive/
}

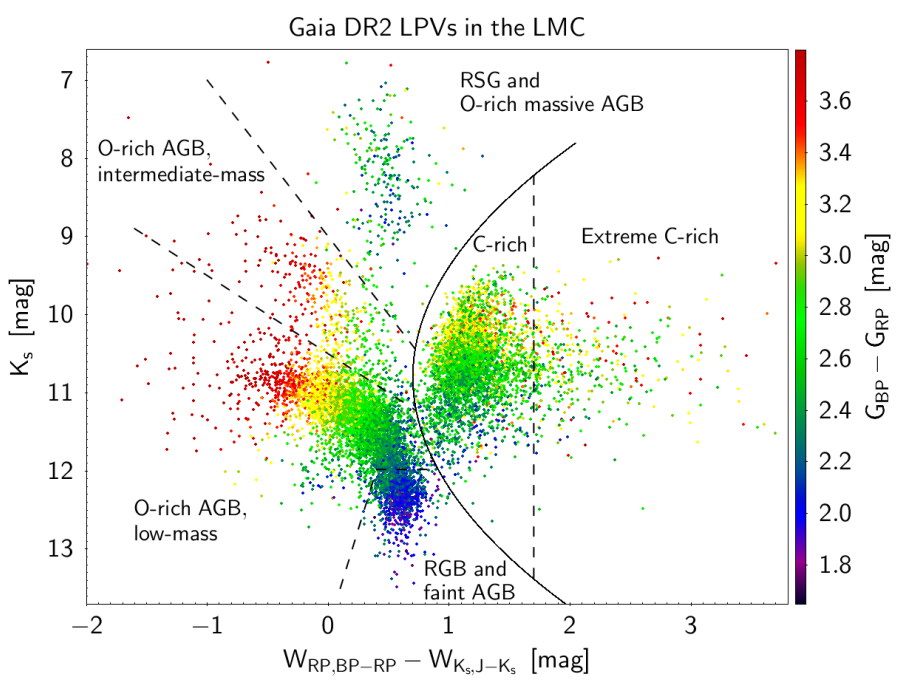

Fig. 1. $\left(W_{\mathrm{RP}, \mathrm{BP}-\mathrm{RP}}-W_{K_{\mathrm{s}}, J-K_{\mathrm{s}}}\right)$ vs. $K_{\mathrm{s}}$ diagram of Gaia DR2 LPVs in the LMC. The markers are colored with $G_{\mathrm{BP}}-G_{\mathrm{RP}}$ according to the color-scale shown on the right of the figure. The solid line delineates O-rich (left of the line) and C-rich (right of the line) stars, and dashed lines delineate sub-groups as indicated in the figure. The definition of the borderlines are given in Appendix A.

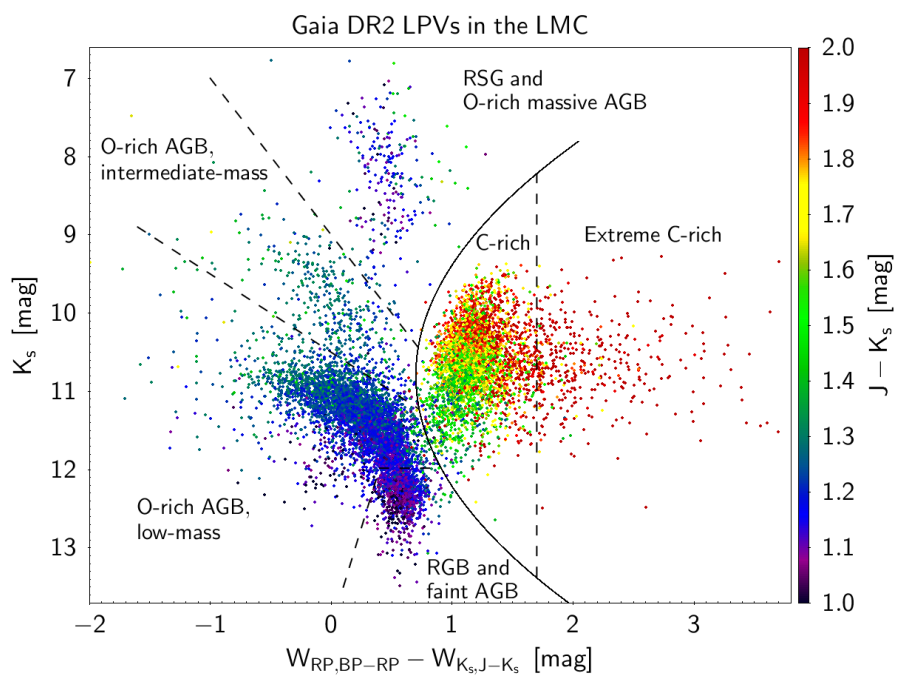

Fig. 2. As in Fig. 1, but with the markers colored with $J-K_{\mathrm{s}}$ according to the color-scale shown on the right of the figure.

decreases to values below $W_{\mathrm{RP}}-W_{K}=0.8 \mathrm{mag}$. Figure 2 , on the other hand, which plots the same diagram but with a color-code related to $J-K_{\mathrm{s}}$, shows that, on the right side of the diagram, stars have an increasing $J-K_{\mathrm{s}}$ color (they also become redder) when $W_{\mathrm{RP}}-W_{K}$ increases to larger values above $W_{\mathrm{RP}}-W_{K}=0.8 \mathrm{mag}$.

\section{Applications of the index $W_{\mathrm{RP}}-W_{K}$}

The $\left(W_{\mathrm{RP}}-W_{K}\right)$ versus $K_{\mathrm{s}}$ diagram turns out to be a very powerful tool to discriminate between various types of AGB stars. This mainly results from two facts. First is the fact that C-rich stars are distinguished from O-rich stars by their redder $J-K_{\mathrm{s}}$ colors (e.g., Marigo et al. 2003) ${ }^{2}$. In the $\left(W_{\mathrm{RP}}-W_{K}\right)$ versus $K_{\mathrm{s}}$ diagram, this implies that $\mathrm{C}$-rich stars will be located on the right side of the diagram, at $W_{\mathrm{RP}}-W_{K} \gtrsim 0.8 \mathrm{mag}$ for the LMC (as

2 The Gaia pipeline did not discriminate between C- and M-type stars in DR2 (Mowlavi et al. 2018). 
confirmed from the location of these stars in the 2MASS colormagnitude diagram, see below), while O-rich stars are expected to be on the left at $W_{\mathrm{RP}}-W_{K} \lesssim 0.8 \mathrm{mag}$. This nice discrimination between $\mathrm{C}$-rich and O-rich chemistry in the diagram is a consequence of distinct molecular absorption features present in the spectra of these two types of star (see Appendix A for more details). The second fact that allows to discriminate various types of AGB stars in the diagram is related to their specific evolution properties as a function of their initial stellar mass, $M_{i}$. Schematically, AGB stars evolve to C-rich stars only for initial stellar masses above a given threshold ${ }^{3}\left(M_{i} \gtrsim 1.45 M_{\odot}\right.$ at $Z=0.006$; see e.g., Marigo et al. 2013). Intermediate-mass AGB stars $\left(M_{i} \gtrsim 3.0-3.5 M_{\odot}\right.$ at $Z=0.006$, depending on model details), on the other hand, generally do not become C-stars due to mass loss and/or hot-bottom burning (HBB). These different types of AGB stars are therefore expected to populate distinct regions in the $\left(W_{\mathrm{RP}}-W_{K}\right)$ versus $K_{\mathrm{s}}$ diagram.

A total of six groups are identified in the $\left(W_{\mathrm{RP}}-W_{K}\right)$ versus $K_{\mathrm{s}}$ diagram, as shown in Figs. 1 and 2. Of these groups, four consist of O-rich stars, located at the left of the solid line on the diagram, while the remaining two groups on the right of the solid line contain the C-rich stars. The separation of C-rich stars into two distinct groups reflects the drop in concentration of stars beyond $W_{\mathrm{RP}}-W_{K} \sim 1.7 \mathrm{mag}$ (see discussion below). Throughout the remainder of this Letter, we refer to these objects as C-rich and extreme C-rich stars, respectively. The term "extreme" was originally introduced by Blum et al. (2006) in their Spitzer SAGE survey of the LMC to identify a group of very red objects in near-mid infrared CMDs, interpreted mostly as dustenshrouded C-rich stars. It should be clear that our criterion for extreme C-rich stars is not the same, but distinguishes a subsample of the extreme objects in the original definition.

The identification of subgroups among the O-rich M-type stars follows the structures suggested in Figs. 1 and 2. The tip of the RGB is located close to $K_{\mathrm{s}}=12$ in the LMC (Cioni et al. 2000). We therefore assume that the stars in the group "RGB and faint AGB" are primarily tip-RGB stars or stars on the early AGB. For the remaining, brighter, O-rich stars, three subgroups are distinguishable in the diagram, as delineated by the dashed lines on the left of the solid line in the two figures. Comparison with model predictions, discussed below, reveals that they are associated with low-mass, intermediate-mass, and massive O-rich AGB stars, respectively.

In order to interpret the observed $\left(W_{\mathrm{RP}}-W_{K}\right)$ versus $K_{\mathrm{s}}$ diagram, we compute stellar population synthesis models of the LMC that take into account the stellar and galactic properties of the Cloud (see Appendix B for details). The result of the simulation is shown in Fig. 3, with the points color-coded according to the initial mass of the simulated stars. The simulated diagram (Fig. 3) shows a similar structure as the observed one (Fig. 1). In particular the segregation of stars in the various branches is well recovered by models. The color-coding clearly indicates that the various groups visible in Fig. 1 can be directly related to various mass ranges (for further comparisons, see Fig. B.1 of the Appendix that nicely associates the branches visible in the diagram to stellar evolution tracks of various masses).

The comparison of the observed (Figs. 1 and 2) and simulated (Fig. 3) diagrams confirms that ${ }^{4}$ :

\footnotetext{
The mass limit for the formation of C-rich stars significantly depends on model details, in particular the third dredge-up efficiency, and varies from author to author. Here we report the results from our ongoing calibration of the TP-AGB phase.

4 The mass limits given in this Letter for the different groups are derived from our LMC simulations.
}

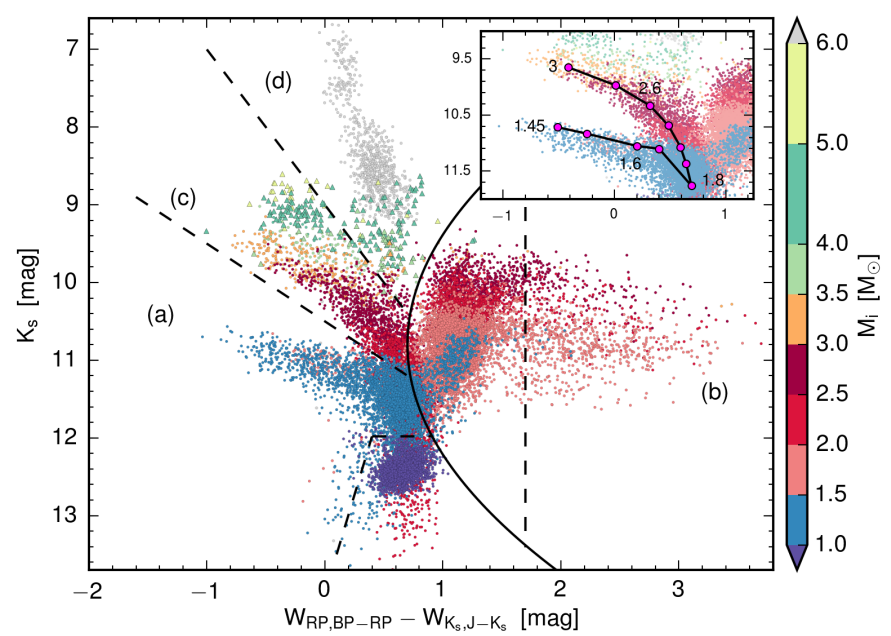

Fig. 3. Synthetic stellar populations for the LMC, color-coded according to the initial stellar mass. The simulation is obtained with the TRILEGAL code (Girardi et al. 2005) and stellar isochrones that include a detailed description of the AGB phase (Marigo et al. 2013, 2017). Suitable selection criteria for LPVs are applied, based on new pulsation models (Trabucchi et al. 2017). Letters a, b, c, and d identify the main branches of evolved stellar populations. In the inset, the curve connects the stages immediately before stars turn to C-stars at varying initial mass in the range from 1.45 to $3 M_{\odot}$ (a few values in $M_{\odot}$ are indicated; the initial metallicity is $Z=0.006$ ). Triangles are used to mark AGB stars with HBB. See text and Appendix B for further explanation. The dashed and solid lines correspond to the same empirical boundaries as in Fig. 1.

- the group "O-rich AGB, low-mass" in Fig. 1 mainly contains low-mass O-rich stars during the early-AGB and thermally pulsing AGB (TP-AGB) phases, with initial masses from $\sim 0.9 M_{\odot}$ to $\sim 1.4 M_{\odot}$ (branch (a) in Fig. 3). These stars do not become carbon stars (in these calculations) and extend to the negative $W_{\mathrm{RP}}-W_{K}$ values which they reach at the end of their AGB evolution. A small number of stars in the mass range $\sim 1.4 M_{\odot}$ to $\sim 1.8 M_{\odot}$ lie on the bright side of branch (a) when they are in the evolutionary stage just prior to becoming carbon stars;

- the group "C-rich" in Fig. 1 contains carbon stars $(\mathrm{C} / \mathrm{O}>1$, branch (b)) with distinct $J-K_{\mathrm{s}}$ colors, see Fig. 3 . In our simulation of the LMC, they are characterized by initial masses from $\sim 1.4 M_{\odot}$ to $\sim 3.2 M_{\odot}$;

- the group "Extreme C-rich" (C-stars with very large $J-K_{\mathrm{s}}$ colors) in Fig. 1 is an extension of the group of C-rich stars in branch (b). In the simulation they are characterized by strong mass loss driven by radiation pressure on carbonaceous dust grains. The relatively low concentration of such objects in the observed diagram is mainly due to the short timescales towards the termination of the AGB phase.

- the group "O-rich AGB, intermediate-mass" in Fig. 1 (branch (c)) actually contains two kinds of star. On the one hand, the faint edge of the branch is populated by $M_{i} \gtrsim 2 M_{\odot}$ to $\sim 3.2 M_{\odot}$ stars that are still O-rich (stars between $\sim 1.8 M_{\odot}$ and $\sim 2 M_{\odot}$ do not move into branch (c) before becoming C-rich). They will move to branch (b) when they turn into C-stars later along the TP-AGB phase. The bright edge of the branch, on the other hand, hosts intermediate-mass stars that never become C-stars, with $M_{i}$ from $\sim 3.2 M_{\odot}$ to $\sim 6 M_{\odot}$. These include AGB stars that experience HBB (see Fig.3). We note that during the very last, extremely short-lived, evolutionary stages characterized by very high mass-loss rates 


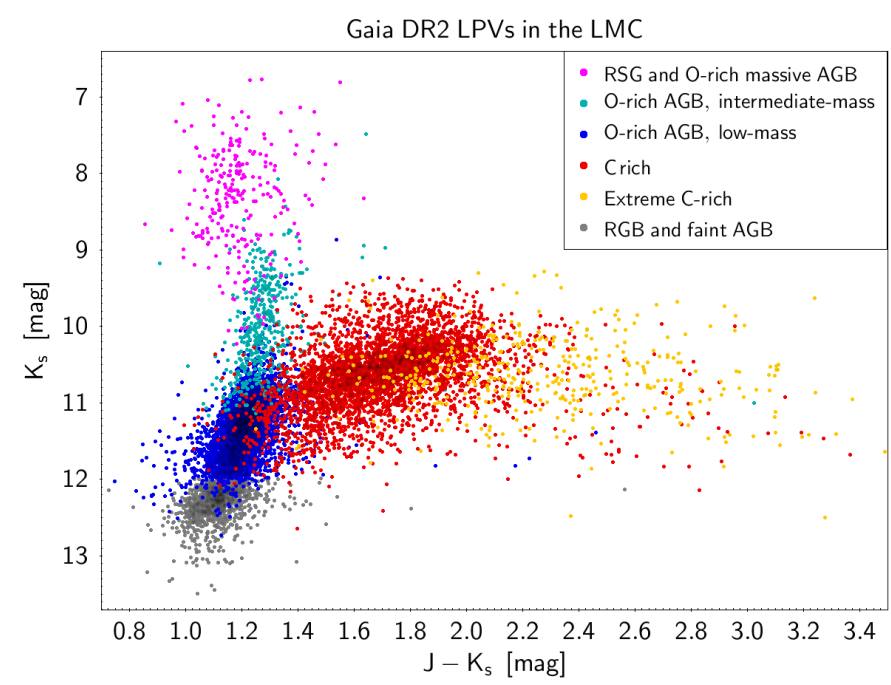

Fig. 4. 2MASS color-magnitude diagram $\left(J-K_{\mathrm{s}}\right)$ versus $K_{\mathrm{s}}$ of Gaia DR2 LPVs in the LMC. The color of the symbols are related to the groups of stars identified in Fig. 1 according to the color-coding written in the inset. The axis ranges have been limited for better visibility.

and efficient dust production, these O-rich intermediate-mass AGB stars can move into the right part of the diagram slightly contaminating the group of extreme $\mathrm{C}$-rich stars. For further discussion we refer to Appendix B;

- the paucity of stars between branch (a) (low-mass O-rich) and branch (b) (intermediate-mass O-rich) reflects the evolution prior to the AGB, besides the properties of the third dredge-up. To help the analysis, in the inset of Fig. 3 we show the predicted location of stars with initial masses in the range $\sim 1.4 M_{\odot}$ to $\sim 3.0 M_{\odot}$ at the stage just before becoming C-stars at the next thermal pulse. As is evident, the curve contours the gap between branches (a) and (c). The fainter section of the curve is drawn by low-mass stars that developed an electron degenerate He-core after the main sequence $\left(\sim 1.4 M_{\odot}\right.$ to $\left.\sim 1.8 M_{\odot}\right)$, while the brighter section is traced by intermediate-mass stars that avoided core degeneracy $\left(\sim 1.8 M_{\odot}\right.$ to $\left.\sim 3 M_{\odot}\right)$. Stars at the transition between the low- and intermediate-mass classes, that is, with $M_{i} \simeq 1.8 M_{\odot}$ (see inset of Fig. 3), ignite He at the smallest core mass of all, and therefore also have the lowest luminosity. With the current TP-AGB models, they also have the faintest subsequent transition luminosity to the C-star domain;

- finally, the group "RSG and O-rich massive AGB" in Fig. 1 is mainly composed of red supergiant stars $\left(M_{i} \gtrsim 8 M_{\odot}\right)$ burning He in their core (branch (d) in Fig. 3). Our models suggest that a small fraction of them, distributed around the faint side of the group, are $M_{i} \simeq 6 M_{\odot}$ to $\sim 8 M_{\odot}$ stars that include super-AGB stars (Siess 2010), as well as massive AGB stars with $M_{i}$ from $\sim 5 M_{\odot}$ to $\sim 6 M_{\odot}$.

The $\left(W_{\mathrm{RP}}-W_{K}\right)$ versus $K_{\mathrm{s}}$ diagram is therefore a powerful tool to identify the different types of AGB stars. Compared to the classical 2MASS color-magnitude diagram, shown in Fig. 4 with the various groups highlighted in color, it provides the power to discriminate AGB stars according to their initial masses in addition to their chemistry.

Finally, we note an interesting feature in the observed $\left(W_{\mathrm{RP}}-\right.$ $W_{K}$ ) versus $K_{\mathrm{s}}$ diagram of the LMC. There seems to be a real gap between the O-rich and C-rich clumps in Figs. 1 and 2. This results from the fast transition between O-rich and C-rich regimes as soon as the $\mathrm{C} / \mathrm{O}$ ratio reaches unity, as a consequence

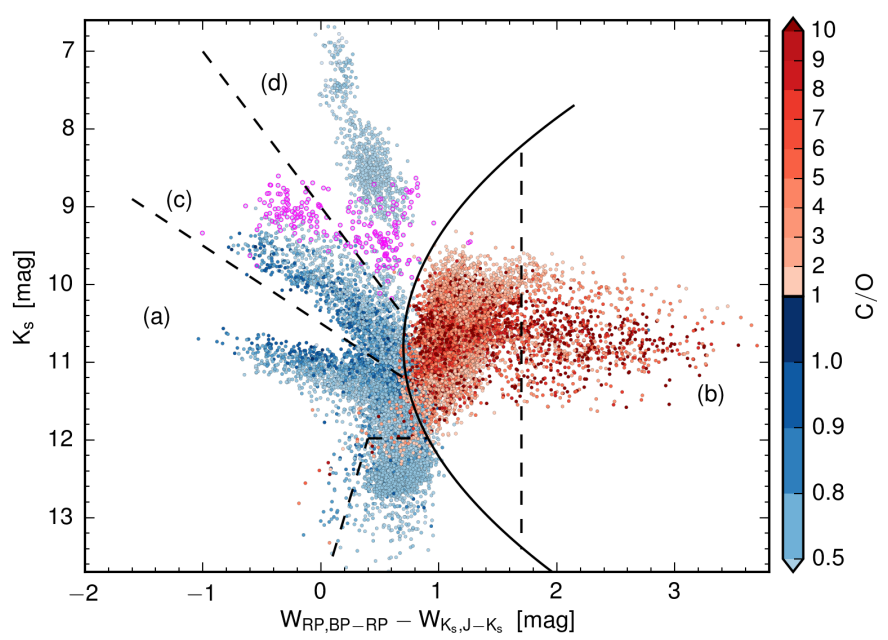

Fig. 5. As in Fig. 3, color-coded according to the $\mathrm{C} / \mathrm{O}$ ratio. AGB stars with HBB are highlighted with magenta circles.

of the abrupt change in the molecular abundance pattern in the atmospheres of these stars (e.g., Marigo \& Aringer 2009). This is clearly visualized in Fig. 5 where we show the simulated $W_{\mathrm{RP}}-W_{K}$ versus $K_{\mathrm{S}}$ of the LMC, color-coded according to the predicted $\mathrm{C} / \mathrm{O}$ ratio. We note that the upper part of branch (a) and the lower part of branch (c) contain O-rich stars with values of the $\mathrm{C} / \mathrm{O}$ close to one (darker blue points in Fig. 5). These should correspond to stars of spectral type S. HBB stars on the bright part of the diagram are characterized by low $\mathrm{C} / \mathrm{O}$ ratios, $N$-enhancement, and low ${ }^{12} \mathrm{C} /{ }^{13} \mathrm{C}$.

\section{Conclusions}

We demonstrate that the new tool for the investigation of stellar populations of AGB-stars presented in this paper offers an excellent possibility to study the large Gaia dataset of evolved stars; it allows to distinguish stars of different mass, chemistry, and evolutionary stage very efficiently. The high diagnostic capability of the new diagram is corroborated with the help of state-of-the-art stellar evolution models.

We plan a more extensive exploration of this tool in the future. As a next step, we are going to use this tool for a similar analysis of other stellar populations and for an investigation on the pulsational behavior of LPVs.

Acknowledgements. We thank the referee for her/his suggestions on sharpening the focus of our Letter. PM, GP, MT acknowledge the support from the ERC Consolidator Grant funding scheme (project STARKEY, G.A. n. 615604).

\section{References}

Aringer, B., Girardi, L., Nowotny, W., Marigo, P., \& Bressan, A. 2016, MNRAS, 457, 3611

Blum, R. D., Mould, J. R., Olsen, K. A., et al. 2006, AJ, 132, 2034

Bressan, A., Marigo, P., Girardi, L., et al. 2012, MNRAS, 427, 127

Cioni, M.-R. L., van der Marel, R. P., Loup, C., \& Habing, H. J. 2000, A\&A, 359,601

Dorda, R., Negueruela, I., González-Fernández, C., \& Tabernero, H. M. 2016, A\&A, 592, A16

Gaia Collaboration (Prusti, T., et al.) 2016, A\&A, 595, A1

Gaia Collaboration (Brown, A. G. A., et al.) 2018, A\&A, 616, A1

Girardi, L., Groenewegen, M. A. T., Hatziminaoglou, E., \& da Costa, L. 2005, A\&A, 436, 895

Gonneau, A., Lançon, A., Trager, S. C., et al. 2017, A\&A, 601, A141

Harris, J., \& Zaritsky, D. 2009, AJ, 138, 1243 
T. Lebzelter et al.: Classification of LPVs using Gaia and 2MASS

Karakas, A. I., \& Lattanzio, J. C. 2014, PASA, 31, e030

Kroupa, P. 2001, MNRAS, 322, 231

Marigo, P., \& Aringer, B. 2009, A\&A, 508, 1539

Marigo, P., Girardi, L., \& Chiosi, C. 2003, A\&A, 403, 225

Marigo, P., Girardi, L., Bressan, A., et al. 2008, A\&A, 482, 883

Marigo, P., Bressan, A., Nanni, A., Girardi, L., \& Pumo, M. L. 2013, MNRAS, 434, 488

Marigo, P., Girardi, L., Bressan, A., et al. 2017, ApJ, 835, 77

Mowlavi, N., Lecoeur-Taïbi, I., \& Lebzelter, T. 2018, A\&A, in press, DOI: $10.1051 / 0004-6361 / 201833366$
Olivier, E. A., \& Wood, P. R. 2005, MNRAS, 362, 1396

Schlegel, D. J., Finkbeiner, D. P., \& Davis, M. 1998, ApJ, 500, 525

Siess, L. 2010, A\&A, 512, A10

Skrutskie, M. F., Cutri, R. M., Stiening, R., et al. 2006, AJ, 131, 1163

Soszynski, I., Udalski, A., Kubiak, M., et al. 2005, Acta Astron., 55, 331

Soszyński, I., Udalski, A., Szymański, M. K., et al. 2009, Acta Astron., 59, 239

Trabucchi, M., Wood, P. R., Montalbán, J., et al. 2017, ApJ, 847, 139

Vassiliadis, E., \& Wood, P. R. 1993, ApJ, 413, 641

Wood, P. R., Alcock, C., Allsman, R. A., et al. 1999, in Asymptotic Giant Branch Stars, eds. T. Le Bertre, A. Lebre, \& C. Waelkens, IAU Symp., 191, 151 
Table A.1. Definition of the different groups identified in the $\left(W_{\mathrm{RP}, \mathrm{BP}-\mathrm{RP}}-W_{K, J-K}\right)$ vs. $K_{\mathrm{s}}$ diagram of Gaia DR2 LPVs (cf. Figs. 1 and 2$)$, valid for the LMC.

\begin{tabular}{ll}
\hline \hline Group candidates & Definition for the LMC \\
\hline O-rich & $W_{\mathrm{RP}}-W_{K} \leq 0.7+0.15 *\left(K_{\mathrm{s}}-10.8\right)^{2}$ \\
- Faint AGB and RGB & O-rich and $K_{\mathrm{s}} \geq 11.98$ and $K_{\mathrm{s}} \geq 13.5-5.067 *\left(W_{\mathrm{RP}}-W_{K}-0.1\right)$ \\
- Low-mass AGB & O-rich and $K_{\mathrm{s}}>10.5+\left(W_{\mathrm{RP}}-W_{K}\right)$ and $\left[K_{\mathrm{s}}<11.98\right.$ or $\left.K_{\mathrm{s}}<13.5-5.067 *\left(W_{\mathrm{RP}}-W_{K}-0.1\right)\right]$ \\
- Intermediate-mass AGB & O-rich and $K_{\mathrm{s}}>9+2 *\left(W_{\mathrm{RP}}-W_{K}\right)$ and $K_{\mathrm{s}} \leq 10.5+\left(W_{\mathrm{RP}}-W_{K}\right)$ \\
- Massive AGB and RSG & O-rich and $K_{\mathrm{s}} \leq 9+2 *\left(W_{\mathrm{RP}}-W_{K}\right)$ \\
\hline C-rich & $W_{\mathrm{RP}}-W_{K}>0.7+0.15 *\left(K_{\mathrm{s}}-10.8\right)^{2}$ \\
- Non extreme C-star & C-rich and $W_{\mathrm{RP}}-W_{K} \leq 1.7$ \\
- Extreme C-star & C-rich and $W_{\mathrm{RP}}-W_{K}>1.7$ \\
\hline
\end{tabular}

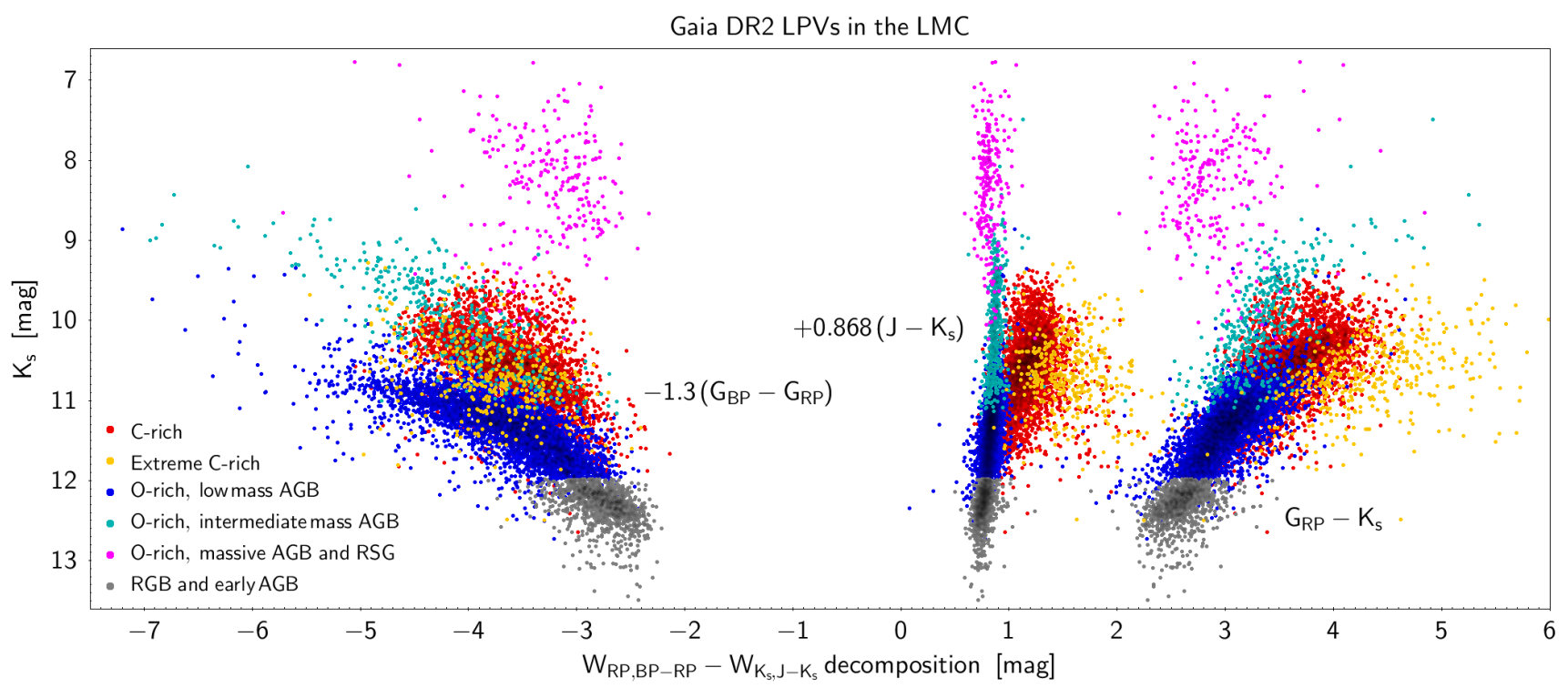

Fig. A.1. $W_{\mathrm{RP}}-W_{K}$ index decomposed into its three contributing colors. The points with negative values on the horizontal axis represent the term $-1.3\left(G_{\mathrm{BP}}-G_{\mathrm{RP}}\right)$, the points between 0 and $\approx 2$ on the horizontal axis represent the term $+0.686\left(J-K_{\mathrm{s}}\right)$ while the points more positive than $\approx 2$ represent the term $G_{\mathrm{RP}}-K_{\mathrm{S}}$. Different stellar groups are marked with different colors as indicated in the lower-left corner of the figure. See text for a detailed description.

\section{Appendix A: A new diagram for distinguishing various groups of stars on the AGB}

In Sect. 2, we present a new diagram to study stars on the upper giant branch. The diagram uses a combination of two Wesenheit functions for the $x$-axis and the $K_{\mathrm{s}}$-band brightness for the $y$-axis. As shown in Fig. 1, six distinct groups of red giants have been identified. The definition of the borders of the groups in this diagram is given in Table A.1. In this appendix, we further elaborate on the newly introduced $W_{\mathrm{RP}}$ Wesenheit function and $W_{\mathrm{RP}}-W_{K}$ index.

The index $W_{\mathrm{RP}}-W_{K}$ actually combines three colors, and cannot be interpreted simply as a color itself. Instead, objects are getting redder both towards lower and higher values of that index with the bluest object being found for the LMC, close to a value of 0.8 .

To understand why this diagram can be used to disentangle various groups on the upper giant branch, we explored the three color indices that enter $W_{\mathrm{RP}}-W_{K}$ separately. Figure A.1 shows the running of each of the three colors individually. We note that $G_{\mathrm{BP}}-G_{\mathrm{RP}}$ enters this plot with a negative sign, since it is subtracted in the combined formula. The sum of the three components as plotted here gives $W_{\mathrm{RP}}-W_{K}$.

For the low-mass O-rich stars (blue dots), the $W_{\mathrm{RP}}-W_{K}$ value is mainly determined by $G_{\mathrm{BP}}-G_{\mathrm{RP}}$ plus a contribution from
$G_{\mathrm{RP}}-K_{\mathrm{s}} . J-K_{\mathrm{s}}$ adds an almost constant value only. In the BP $(\sim 330-680 \mathrm{~nm})$ and RP $(\sim 640-1050 \mathrm{~nm})$ wavelengths range, the spectra of O-rich stars are dominated by molecular bands from $\mathrm{TiO}, \mathrm{VO}, \mathrm{ZrO}$, and VO. All these molecular bands increase in strength with decreasing temperature. The strong effect we see in this color stems from the fact that the flux at the longward end $(\sim 1 \mu \mathrm{m})$ of the RP filter is only mildly affected by molecular absorption. This is nicely illustrated in Fig. 1 of Aringer et al. (2016) showing model spectra in this wavelength range for various values of $T_{\text {eff }}$. Accordingly, the change in $W_{\mathrm{RP}}-W_{K}$ from 0.5 to -1.5 seen in Fig. 1 indicates a change towards lower temperature.

A very similar situation is seen for the intermediate-mass O-rich stars (cyan points in Fig. A.1), which is not surprising since the same molecules will affect the spectrum. Massive AGB stars and supergiants (magenta points), on the other hand, show smaller changes in all three colors compared to the other groups. This is in agreement with red supergiants exhibiting on the average higher $T_{\text {eff }}$ values (Dorda et al. 2016) compared to low- and intermediate-mass AGB stars. As a consequence, this group is found at an almost constant value in $W_{\mathrm{RP}}-W_{K}$ in Fig. 1.

For C-stars (red points), the $W_{\mathrm{RP}}-W_{K}$ value is almost identical to $J-K_{\mathrm{s}}$. The other two colors cancel each other out since they have almost exactly the same value. $G_{\mathrm{BP}}-G_{\mathrm{RP}}$ is dominated by $\mathrm{CN}$ and $\mathrm{C}_{2}$ bands in this case, while the $K_{\mathrm{s}}$ band is only mildly affected by $\mathrm{C}_{2}, \mathrm{CN}$, and $\mathrm{CO}$ (compare Fig. 3 in Gonneau et al. 2017). 


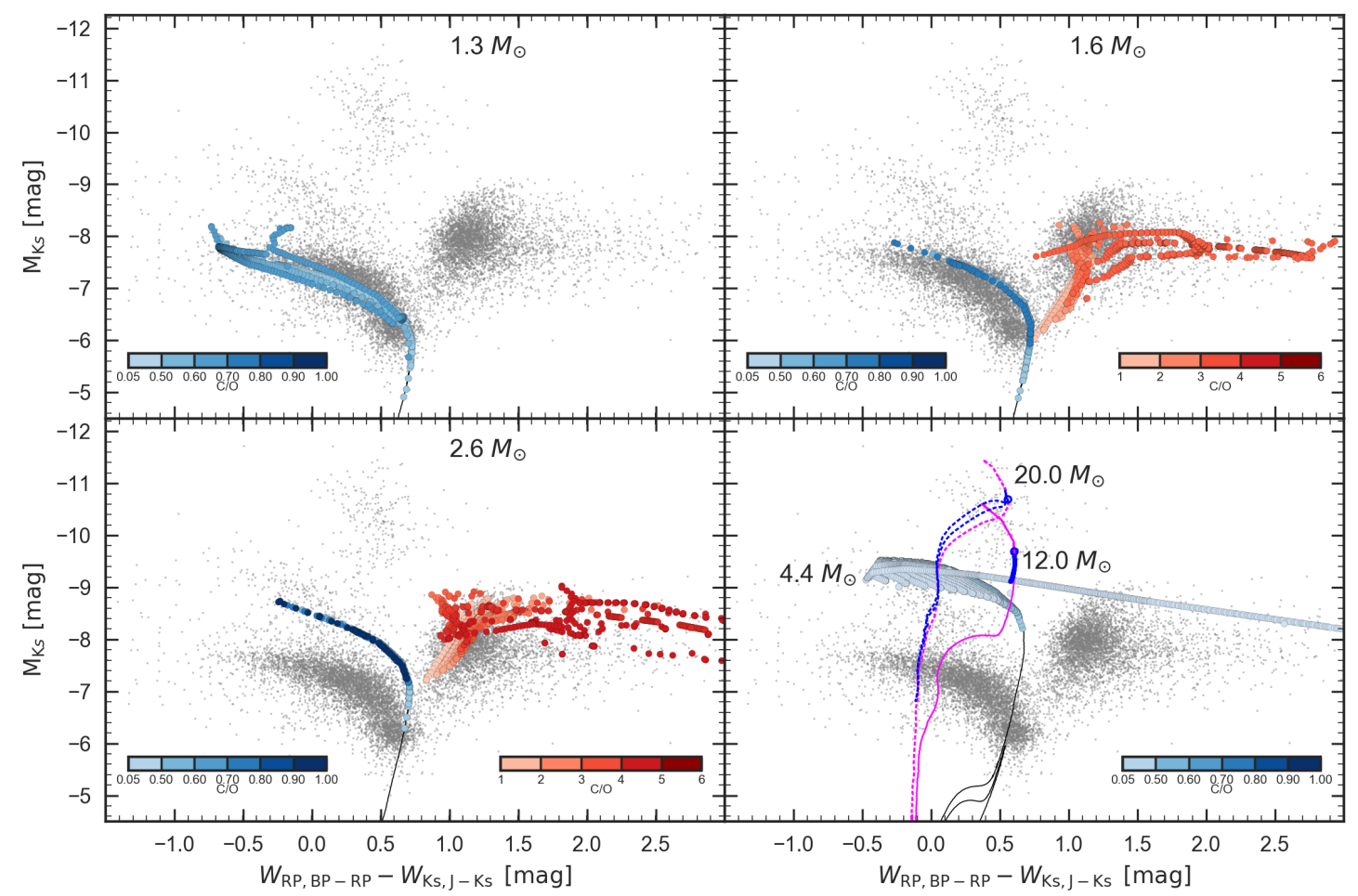

Fig. B.1. Location of stellar evolution tracks computed with the PARSEC and COLIBRI codes (Bressan et al. 2012; Marigo et al. 2013) for a few selected values of the initial mass (as indicated). The initial metallicity is $Z=0.006$ for the tracks with $M_{i}=1.3,1.6,2.6$, and $4.4 M_{\odot}$, and $Z=0.008$ for the massive ones with $M_{i}=12 M_{\odot}$ (solid line) and $20 M_{\odot}$ (dashed line). The TP-AGB phase is color-coded according to the surface $\mathrm{C} / \mathrm{O}$ ratio, while the previous evolution is shown with a black line. In the right-bottom panel the visible sections of the massive star tracks include part of the $\mathrm{H}$-shell burning phase, the core-helium burning phase in blue (a blue loop is visible for the $20 M_{\odot}$ models), and the stages immediately preceding the ignition of carbon in the core. The circles mark the beginning of the core-helium-burning phases in the two massive stars.

\section{Appendix B: Synthetic stellar populations for the LMC: photometry and long-period variables}

\section{B.1. Stellar evolution tracks in the $W_{\mathrm{RP}}-W_{K}$ versus $K_{\mathrm{s}}$ diagram}

During their evolution on the AGB, stars of low and intermediate mass exhibit strong variations of their stellar parameters including temperature, composition, and luminosity on various timescales. These variations have been extensively studied with the help of stellar evolution models (e.g., Vassiliadis \& Wood 1993; Marigo et al. 2008) because of their significant impact on stellar and galactic evolution (e.g., Karakas \& Lattanzio 2014; Marigo et al. 2017). In this appendix we confront predictions from stellar evolution models with the representation of the AGB population in the LMC provided by the newly introduced $W_{\mathrm{RP}}-W_{K}$ versus $K_{\mathrm{s}}$ diagram.

In Fig. B.1 we follow the evolutionary path of stars of four different masses, $1.3 M_{\odot}, 1.6 M_{\odot}, 2.6 M_{\odot}$, and $4.4 M_{\odot}$, and a typical LMC metallicity $(Z=0.006)$ on the background of LMCLPVs identified from Gaia DR2 data. The four masses are good representatives of the mass ranges to which we related the various branches in Figs. 1 and 3. The thermal pulses are clearly visible in all four cases. The locations of these tracks nicely confirm the picture in Sect. 2.

The TP-AGB evolution of the $1.3 M_{\odot}$ model well covers the lowest branch on the left branch (a) of Fig. 3), and is representative of low-mass stars that do not become carbon stars.
The 1.6 $M_{\odot}$ and $2.6 M_{\odot}$ models spend part of their AGB evolution as $\mathrm{O}$-rich, and later become carbon stars as a consequence of a few third dredge-up events. It is interesting to note that as long as the surface $\mathrm{C} / \mathrm{O}$ remains below unity during their TPAGB phase, the two tracks evolve along the distinct leftward branches of the diagram: the low-mass $1.6 M_{\odot}$ model is found at fainter magnitudes, and the intermediate-mass $2.6 M_{\odot}$ model runs brighter branches (a) and (c) of Fig. 3, respectively). The reader is referred to Sect. 2 for the interpretation of the observed gap between the two sequences.

The maximum leftward excursion of both tracks (most negative $W_{\mathrm{RP}}-W_{K}$ value) corresponds to the stage (typically with $\mathrm{C} / \mathrm{O} \simeq 0.8-0.9$ ) immediately before the models become $\mathrm{C}$-rich at the next thermal pulse. When such transition occurs, the newly born C-rich models jump on the rightward branch (sequence b) in Fig. 3), where they evolve until the end of the AGB. The final, short-lived phases are characterized by significant mass loss and efficient dust formation, which drive the models to populate the region of the so-called extreme $\mathrm{C}$-stars (with large $J-K_{\mathrm{S}}$ colors).

The $4.4 M_{\odot}$ model represents the case of a star that experiences HBB. While the brightest portion of the early-AGB takes place at almost constant $W_{\mathrm{RP}}-W_{K}$, the subsequent TP-AGB evolution develops on the brighter leftward branch sequence (c) in Fig. 3). We also expect that HBB stars populate the region spread around the base of the almost vertical branch where the RSG stars are found branch (d) in Fig. 3). The last steps in the evolution on the AGB, when the star moves to high values in $J-K_{\mathrm{s}}$ due to heavy mass loss, occur on very short time scales for these objects. 
To complete the picture of the various mass domains, we plot the evolutionary tracks of a $12 M_{\odot}$ and $20 M_{\odot}$ models with initial metallicity $Z=0.008$ (bottom right panel of Fig. B.1). The two tracks populate the lower and the upper part of branch (d) (see Fig. 3).

\section{B.2. Synthetic stellar populations}

We computed a detailed simulation for the stellar populations of the LMC with the aid of the TRILEGAL code (Girardi et al. 2005). To this aim, we adopted the results from Harris \& Zaritsky (2009) to describe the spatially resolved star formation history, age-metallicity relation, distance and reddening for about 400 regions across the LMC. We adopt a Kroupa IMF (Kroupa 2001) and we calibrated the total mass in stars of each sub-field to reproduce the RGB star counts. The total mass in stars of the simulation is $\approx 2.3 \times 10^{9} M_{\odot}$. Stellar isochrones, based on extended grids of PARSEC and COLIBRI evolutionary tracks (Bressan et al. 2012; Marigo et al. 2013), are described in Marigo et al. (2017). They include the effect on simulated photometry due to circumstellar dust around mass-losing AGB stars. Evolution along the thermally pulsing AGB is followed up to the complete ejection of the envelope by stellar winds and accounts for the formation of carbon stars due to the third dredge-up, as well as the occurrence of HBB in the most massive TP-AGB stars (with initial masses $>4 M_{\odot}$ at the relevant metallicities). In this work, we used a set of available TP-AGB tracks calibrated to reproduce star counts and luminosity functions for AGB stars in the SMC (Pastorelli et al., in prep.).

The simulation is complete down to $G=20 \mathrm{mag}$. In Gaia DR2, a total number of 11022 LPVs in the LMC have magnitudes brighter than $K_{\mathrm{s}}=13.7 \mathrm{mag}$ and $G_{\mathrm{RP}}=17.5$. Adopting these limits, our LMC simulation predicts a much higher number of stars, that is, about $1.69 \times 10^{5}$.

The excess of simulated stars corresponds to red giants with variability amplitudes that are too low for them to be detected as variables. These need to be excluded from the simulation for a consistent comparison with observations. In principle, this would require estimation of variability amplitudes for the stars in the simulation. However, a simple relation between pulsation amplitudes and global stellar parameters is currently unavailable, meaning that direct computations with non-linear pulsation models would be necessary. The main obstacles in the use of non-linear models are that: (1) to reproduce observations, they include free parameters, which lack a proper calibration as a function of global stellar parameters (see, e.g., Olivier \& Wood 2005, and references therein), and (2) they are generally time-consuming, and therefore cannot be readily implemented in present synthetic populations.

For these reasons, we follow a different approach, based on the results of linear pulsation models. To begin with, we exploit the fact that the dominant mode of pulsation in LPVs shifts towards lower radial orders as they evolve, with the fundamental mode being the last one to become dominant, right after the first overtone mode. All stars in our observed sample are pulsating either in the first overtone or in the fundamental mode (Lebzelter et al., in prep.), that is, all of them have evolved to the point at which the first overtone mode has become dominant, or past it. To determine which simulated stars have gone through that point, we make use of the fact that, for overtone modes, growth rates are a good proxy for variability amplitudes (Trabucchi et al. 2017). To a first approximation, they depend only on the ratio between the dynamical frequency and the acoustic cut-off frequency of the star, proportional to $\tilde{v}=\left(R T_{\text {eff }} / M\right)^{1 / 2}$ (Trabucchi et al., in prep.). The dependence is monotonic, and follows a functional form that is similar in the range of parameters corresponding to typical AGB stars, for which one finds $4 \lesssim \tilde{v} \lesssim 18$. This allows to formulate a selection criterion in terms of global stellar parameters: we keep only simulated stars for which

$\tilde{v}=\left(R T_{\text {eff }} / M\right)^{1 / 2} \gtrsim \tilde{v}_{\text {min }} \simeq 10$,

where the threshold on the right-hand side is estimated from linear pulsation models. The exact value depends on the relation between amplitudes and growth rates, which has not yet been derived. However, this does not affect our results. We find that the morphology of the observed $\left(W_{\mathrm{RP}}-W_{K}\right)$ versus $K_{\mathrm{S}}$ diagram is reasonably well reproduced assuming $\tilde{v}_{\min } \simeq 8-10$, depending slightly on mass and chemical type. Being based on pulsation models appropriate for AGB stars, the above considerations cannot be applied to RSGs on branch (d), which are experiencing the core He-burning phase. Therefore, no selection is applied to those stars. 